\title{
Modelo matemático 1D da dinâmica de um glioma com coeficiente de difusão descontínuo e capacidade de carga variável
}

\author{
1D mathematical model of the dynamics of a glioma with discontinuous diffusion coefficient \\ and variable carrying capacity
}

$\begin{array}{r}\text { Gabriel Carlos Pena da Silva } \\ \text { Universidade Federal de São João del-Rei (UFSJ), Engenharia Elétrica, São João del-Rei, MG, } \\ \text { Brasil }\end{array}$
https://orcid.org/0000-0003-1155-3594, gabrielcarlospena8@hotmail.com

Jorge Andrés Julca Avila

Universidade Federal de São João del-Rei (UFSJ), Departamento de Matemática e Estatística, São João del-Rei, MG, Brasil https://orcid.org/0000-0002-8118-8425, avila_jaj@ufsj.edu.br

\section{Informações do Artigo}

\section{Como citar este artigo}

SILVA, G. C. P. da; AVILA, J. A. J. Modelo matemático 1D da dinâmica de um glioma com coeficiente de difusão descontínuo e capacidade de carga variável. REMAT: Revista Eletrônica da Matemática, Bento Gonçalves, RS, v. 6, n. 2, p. e4003, 13 ago. 2020. DOI: https://doi.org/10. 35819/remat2020v6i2id4067

\section{Histórico do Artigo}

Submissão: 3 de abril de 2020.

Aceite: 7 de maio de 2020 .

\section{Palavras-chave}

Oncologia Matemática

Glioma

Equação de Difusão-Reação

Capacidade de Carga Variável

Métodos Numéricos

\section{Resumo}

Neste trabalho, resolveremos numericamente a equação que modela o problema da dinâmica do crescimento de um glioma, com capacidade de carga que varia espacialmente. Devido à natureza difusiva do glioma, o problema é modelado pela Equação de Difusão-Reação (ED-R). Estudaremos o caso unidimensional (1D). A ED-R apresenta um perfil Gaussiano como condição inicial e condição de contorno do tipo Neumman. $O$ microambiente tumoral é uma porção do cérebro, constituída, principalmente, por células do glioma. Ele apresenta três regiões: duas regiões de substâncias cinzentas, localizadas na parte extrema do microambiente, e uma região de substância branca, localizada no meio do microambiente. Dois fatos importantes caracterizam a modelagem desse problema. Primeiro, o coeficiente de difusão é uma função descontínua, e segundo, a capacidade de carga, no modelo de crescimento logístico, é uma função de tipo Hill que depende da variável espacial. O problema é resolvido numericamente pelo método de Crank-Nicolson, e os resultados numéricos apontam diminuição do crescimento tumoral ao considerar-se a capacidade de carga variável. 


\section{Keywords}

Mathematical Oncology

Glioma

Reaction-Diffusion Equation

Variable Carrying Capacity

Numerical Methods

\begin{abstract}
In this work, we will numerically solve the equation that models the problem of the growth dynamics of a glioma, with carrying capacity that varies spatially. Due to the diffusive nature of the glioma, the problem is modeled by the Reaction-Diffusion Equation (RDE). We will study the one-dimensional case (1D). The RDE has a Gaussian profile, as an initial condition, and a boundary condition of the Neumman type. The tumor microenvironment is a portion of the brain, consisting mainly of glioma cells. It has three regions: two regions of gray matter, located in the extreme part of the microenvironment, and a region of white substance, located in the middle of the microenvironment. Two important facts characterize the modeling of this problem. First, the diffusion coefficient is a discontinuous function, and second, the carrying capacity, in the logistic growth model, is a Hill-type function that depends on the spatial variable. The problem is solved numerically by the Crank-Nicolson method, and the numerical results indicate a decrease in tumor growth when considering the variable carrying capacity.
\end{abstract}

\section{Introdução}

Neoplasia literalmente significa "novo crescimento". As células neoplásicas são transformadas porque continuam a se replicar, aparentemente "desatentas" às influências regulatórias que controlam o crescimento celular normal. As neoplasias, portanto, desfrutam de certo grau de autonomia e tendem a aumentar de tamanho independentemente de seu ambiente local (KUMAR; ABBAS; ASTER, 2013).

No uso médico comum, geralmente uma neoplasia é referida como tumor, e o estudo dos tumores é chamado de Oncologia. Os tumores podem ser classificados em (a) Tumor benigno e (b) Tumor maligno. Em (a), Tumor benigno, as células crescem vagarosamente e podem ser removidas completamente do paciente por meio de cirurgia; geralmente o paciente sobrevive. Note-se, porém, que os tumores benignos podem produzir mais do que massas localizadas e, algumas vezes, são responsáveis por doença grave. O (b), Tumor maligno, é referido coletivamente como cânceres, termo derivado da palavra em latim "caranguejo", ou seja, ele adere a qualquer parte onde se agarra e de maneira obstinada, semelhante ao comportamento do caranguejo (KUMAR; ABBAS; ASTER, 2013). Existem duas propriedades que diferenciam os tumores malignos: a invasividade local e a metástase. A invasividade local é o potencial para infiltrar a estrutura anatômica que originou o tumor, assim como os órgãos e tecidos adjacentes. A metástase é a disseminação à distância; essa característica depende da permeabilidade dos vasos linfáticos e sanguíneos às células neoplásicas, o que possibilita que estas ingressem à vasculatura e acedam a órgãos e tecidos distantes (GARCIA; GÓMEZ, 2010). 
Um dos trabalhos pioneiros no ramo da oncologia é devido a Folkman (1976). Ele formula as hipóteses de crescimento de tumores sólidos, divididos em duas fases: avascular e vascular. Já, com relação aos trabalhos sobre oncologia matemática, Chaplain e Sleeman (1990) elaboram um modelo matemático que descreve os fatores de angiogênese tumoral. A partir desses trabalhos, apareceram muitos outros nessa direção. Alguns temas em oncologia matemática são: o crescimento tumoral (DEHGHAN; MOHAMMADI, 2017), invasão de células tumorais (AVILA; LOZADA-CRUZ, 2013), angiogênese (BONILLA et al., 2017), metástase (HARTUNG et al., 2014), entre outros.

O Sistema Nervoso Central (SNC) é formado pelo encéfalo e pela medula espinhal. cérebro, parte fundamental do encéfalo, é composto de duas grandes classes de células, os neurônios e as células gliais. A diferença entre essas células é que a primeira tem a capacidade de enviar sinais uns para outros através de longas distâncias, fato que não acontece com as células gliais. O neurônio divide-se em várias partes: corpo celular, dendritos e axônio. No SNC observa-se as substâncias branca e cinzenta. A substância branca, basicamente, corresponde aos axônios, ao passo que a substância cinzenta corresponde aos corpos celulares. No encéfalo, a substância branca está distribuída no interior, enquanto a substância cinzenta está distribuída no córtex cerebral, que é a camada externa do cérebro.

Segundo o Instituto Nacional de Câncer (INCA, 2019), para o Brasil, a cada ano do triênio 2020-2022, estimam-se 5.870 casos novos de câncer no SNC em homens e 5.220 em mulheres. A maior parte dos tumores do SNC se origina no cérebro, nervos cranianos e meninges. Os gliomas são o tipo histológico mais frequente e representam cerca de $40 \%$ a $60 \%$ de todos os tumores primários do SNC, sendo mais comuns na faixa etária adulta. Em geral, esses tumores são cirurgicamente incuráveis, além de possuírem resistência à radiação e à quimioterapia.

Segundo o Instituto Oncoguia (2018), glioma é um termo geral para um grupo de tumores que se iniciam nas células gliais. Vários tipos de tumores podem ser considerados gliomas, como: o glioblastoma multiforme, astrocitomas, oligodendrogliomas e ependimomas. Cerca de $30 \%$ de todos os tumores cerebrais são gliomas. A maioria dos tumores cerebrais de crescimento rápido e agressivos são os gliomas. As células menores que compõem os gliomas são as células da micróglia. O glioma é a forma mais maligna de tumor cerebral quantificada em termos de taxas líquidas de proliferação e invasão.

Trabalhos numéricos em oncologia matemática, em particular em gliomas, estão sendo desenvolvidos por muitos pesquisadores. Cruywagen et al. (1995) foram os primeiros que modelaram tumores difusivos, mais especificamente, tumores difusivos cerebrais. Swanson, Alvord e Murray 
(2000) apresentam um modelo quantitativo para motilidade diferencial de gliomas nas substâncias branca e cinza. Özuğurlu (2015) resolve numericamente a ED-R 1D que modela o crescimento de um glioma com coeficiente de difusão descontínuo e capacidade de carga constante. Com relação à solução numérica e experimental, em modelagem de gliomas, um artigo relevante é devido a Kim, Jeon e Othmer (2017), que elaboram um modelo matemático do papel que desempenha o glioma em um microambiente tumoral. Eles definem um microambiente tumoral como um lugar que possui células tumorais, matriz extracelular, fatores promotores e inibidores de crescimento, nutrientes, quimiocinas e outros tipos de células no tecido estromal.

A capacidade de carga de um tumor é o número máximo de células tumorais que seu microambiente pode suportar em condições normais, ou seja, com os nutrientes disponíveis.

Em problemas de crescimento de glioma difusivo, geralmente, considera-se o microambiente tumoral homogêneo (ou relativamente uniforme) e capacidade de carga constante. Em dinâmica populacional em ecologia, Deangelis et al. (2020) estudaram, em um ambiente heterogêneo, a capacidade de carga dependente da variável espacial.

Neste trabalho, resolveremos numericamente a equação que modela o problema da dinâmica do crescimento de um glioma, com capacidade de carga que varia espacialmente. Devido à natureza difusiva do glioma, o problema é modelado pela Equação de Difusão-Reação (ED-R). Estudaremos o caso unidimensional (1D). A ED-R apresenta um perfil Gaussiano como condição inicial e condição de contorno do tipo Neumman. O microambiente tumoral é uma porção do cérebro, constituída, principalmente, por células do glioma. Ele apresenta três regiões: duas regiões de substâncias cinzentas, localizadas na parte extrema do microambiente, e uma região de substância branca, localizada no meio do microambiente; dessa forma, torna-se heterogêneo. Dois fatos importantes caracterizam a modelagem desse problema. Primeiro, o coeficiente de difusão é uma função descontínua (o que implica considerar discretizações numéricas apropriadas) e, segundo, a capacidade de carga, no modelo de crescimento logístico, é uma função de tipo Hill que depende da variável espacial. O problema é resolvido numericamente pelo método de Crank-Nicolson, e os resultados numéricos apontam diminuição do crescimento tumoral ao considerar-se a capacidade de carga variável.

\section{Formulação matemática do problema}

Nesta seção, estudaremos a equação que governa o problema, o domínio e as condições inicial e de contorno. 


\subsection{Equações governantes}

A Equação Diferencial Parcial (EDP) de $2^{\mathrm{a}}$ ordem, não-linear, que modela o problema da dinâmica de crescimento do glioma, considerando sua natureza difusiva, é a ED-R. Neste trabalho, consideraremos a ED-R 1D, na qual, o termo da taxa líquida de proliferação, regido pelo modelo de crescimento logístico, possui capacidade de carga variável. Essa equação é dada por

Taxa de variação

da concentração de células tumorais

$$
\overbrace{\frac{\partial c}{\partial t}}
$$

Difussão

de células tumorais

$\overbrace{\frac{\partial}{\partial x}\left(D(x) \frac{\partial c}{\partial x}\right)}+$
Taxa líquida

de proliferação de células tumorais

$\overbrace{r c\left(1-\frac{c}{\kappa(x)}\right)}$

onde

$c(x, t)$ : concentração de células do glioma na posição $x$ e no instante de tempo $t$ [células $/ \mathrm{mm}$ ].

$D(x) \quad$ : coeficiente de difusão variável e descontínuo $\left[\mathrm{mm}^{2} / \mathrm{dia}\right]$.

$r \quad$ : taxa de proliferação de células tumorais $[1 / \mathrm{dia}]$.

$\kappa(x) \quad$ : capacidade de carga do glioma, variável [células $/ \mathrm{mm}$ ].

\subsubsection{Coeficiente de difusão}

Segundo Özuğurlu (2015), podemos considerar o coeficiente de difusão dado por

$$
D(x)=\left\{\begin{array}{llll}
D_{g}=0.13, & \text { se } 0 \leqslant x<7.5 & & \text { (região cinza) } \\
D_{w}=0.65, & \text { se } 7.5 \leqslant x \leqslant 42.5 & & \text { (região branca) } \\
D_{g}=0.13, & \text { se } 42.5<x \leqslant 50 & & \text { (região cinza) }
\end{array}\right.
$$

representando, geometricamente, uma onda retangular. O gráfico dessa função é apresentada na Figura 1, sendo $x$ a posição no microambiente tumoral de comprimento $50 \mathrm{~mm}$.

\subsubsection{Capacidade de Carga Variável}

Segundo Deangelis et al. (2020), a análise de modelos de difusão-reação de populações em meio heterogêneo mostrou que, quando a taxa de crescimento máximo e a capacidade de carga de um modelo de crescimento logístico variam espacialmente, existem condições para as quais o tamanho total da população em equilíbrio pode exceder à população total. Além disso, o tamanho total da população, em um sistema heterogêneo com difusão, pode exceder o tamanho de um sistema sem difusão. Dessa forma, podemos considerar a capacidade de carga dependente da variável espacial 
Figura 1 - Gráfico do coeficiente de difusão, $D(x)$, sendo $x$ a posição no microambiente tumoral de comprimento $50 \mathrm{~mm}$.

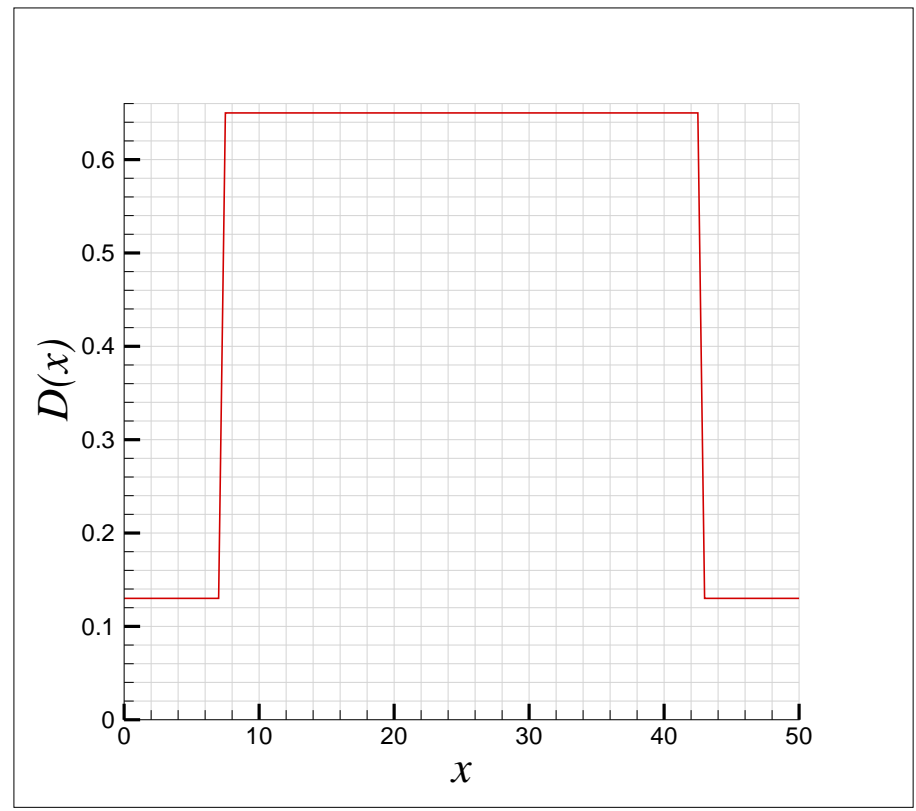

Fonte: Elaboração dos autores (2020).

$x$. Definimos a função capacidade carga do glioma por uma função de Hill

$$
\kappa(x)=\left(c_{\max }-a\right)\left[\frac{17,5^{50}}{17,5^{50}+(x-25)^{50}}\right]+a
$$

onde $c_{\max }=62,5$ é a capacidade de carga máxima, obtida de Özuğurlu (2015), e $a$ é uma constante real, $0 \leqslant a \leqslant c_{\max }$. Note que, se $a=c_{\max }$, a capacidade de carga $\kappa(x)$ é constante e toma o seu valor máximo. Considerou-se, no microambiente tumoral, que os valores da capacidade de carga do glioma, nas regiões da substância cinzenta, seja menor que na região da substância branca. $O$ gráfico da função capacidade de carga do glioma para os casos particulares $a=0, a=10$ e $a=20$, é apresentado na Figura2.

\subsection{Domínio do problema}

Seja $X=\left(x_{1}, x_{2}\right)$ um intervalo aberto. Então, $\bar{X}=\left[x_{1}, x_{2}\right]$ é o fecho de $X$. Por outro lado, $\bar{X}^{*}=\bar{X}-\left\{x_{1}\right\}=\left(x_{1}, x_{2}\right]$ e $\partial \bar{X}=\left\{x_{1}, x_{2}\right\}$ é a fronteira de $\bar{X}$.

O domínio do problema é o microambiente tumoral 1D. Ele é definido por $\Omega=(0, L)$, sendo $L$ o comprimento desse microambiente. A variável espacial $x \in \Omega$ e, a variável temporal $t \in I=$ $\left(0, T_{\max }\right)$, onde $T_{\max }$ é o tempo máximo que se precisa para atingir o regime permanente. 
Figura 2 - Gráfico da função capacidade de carga do glioma, $\kappa(x)$, sendo $x$ a posição no microambiente tumoral de comprimento $50 \mathrm{~mm}$. Foram plotados para diferentes valores: $a=0, a=10$ e $a=20$.

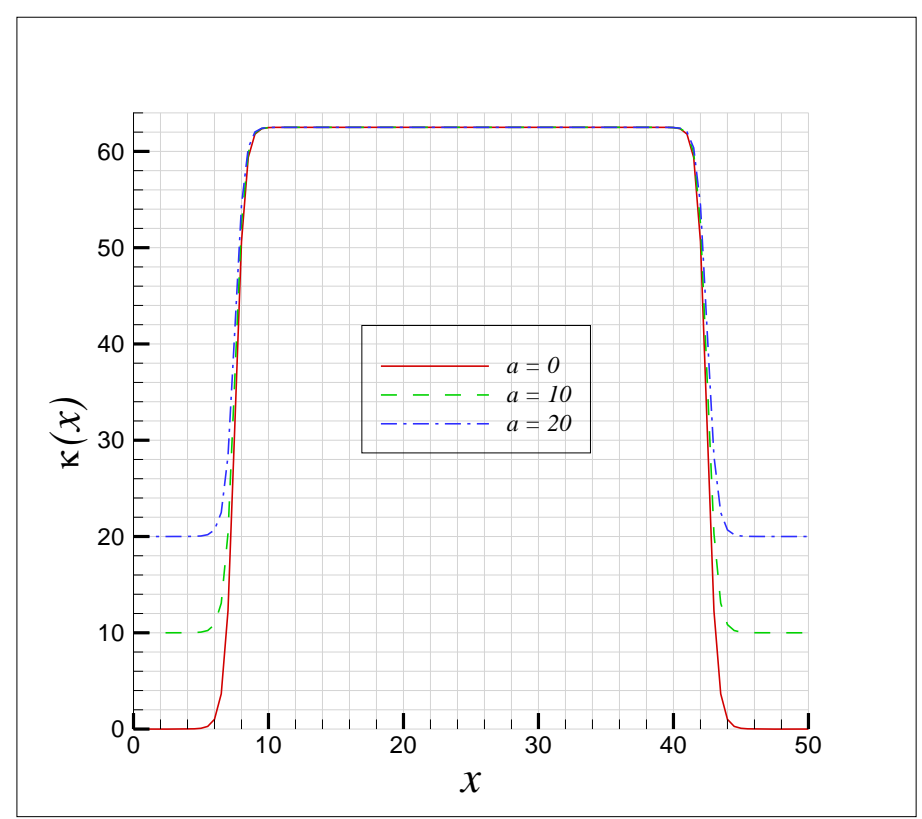

Fonte: Elaboração dos autores (2020).

O domínio espaço-temporal do problema é definido pelo retângulo $Q=\bar{\Omega} \times \bar{I}=[0, L] \times$ $\left[0, T_{\max }\right]$. A função $c(x, t)$ depende de duas variáveis: uma espacial e a outra temporal, isto é, $(x, t) \in \bar{\Omega} \times \bar{I}$.

\subsection{Condições inicial e de contorno}

\subsubsection{Condição inicial}

A condição inicial segue um perfil gaussiano, conforme Özuğurlu (2015),

$$
c_{0}(x)=c(x, 0)=\frac{1}{\sqrt{2 \pi} \epsilon} \mathrm{e}^{-\frac{1}{2}\left(\frac{x-x_{c}}{\epsilon}\right)^{2}}, \quad 0<x<50
$$

onde $x_{c}=25 \mathrm{~mm} \mathrm{e} \epsilon=0,01$. O gráfico dessa função é apresentada na Figura 3 .

\subsubsection{Condição de contorno}

Foram consideradas as condições de contorno do tipo Neumann, conforme Özuğurlu (2015),

$$
\frac{\partial c(0, t)}{\partial x}=0 \quad \text { e } \frac{\partial c(50, t)}{\partial x}=0
$$


Figura 3 - Gráfico de condição inicial, $c_{0}(x)=c(x, 0)$, sendo $x$ a posição no microambiente tumoral.

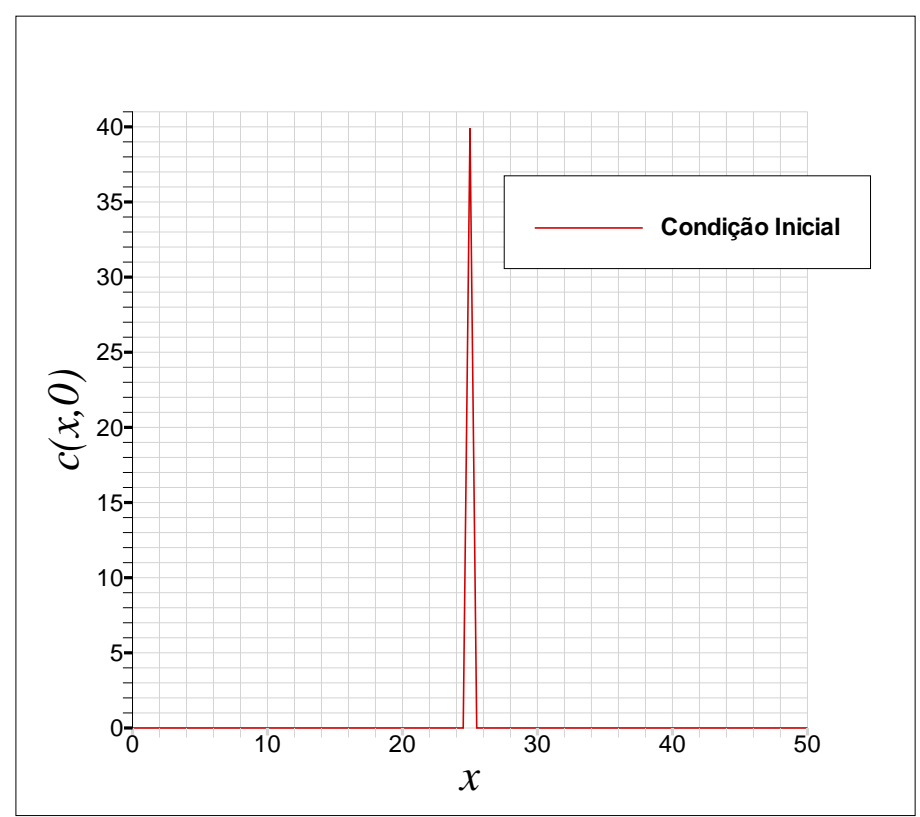

Fonte: Elaboração dos autores (2020).

\section{Formulação numérica do problema}

Apresentaremos, nesta seção, a discretização da equação que modela o problema, e o método de Crank-Nicolson.

\subsection{Discretização do domínio}

Serão definidas as discretizações espacial, temporal e espaço-temporal do domínio do problema.

\subsubsection{Discretização espacial}

Defina-se:

$$
\begin{aligned}
h & =(L-0) /(N+1), \quad x_{i}=0+i h, \quad i=0,1,2, \ldots, N+1 \\
\bar{\Omega}_{h} & =\left\{x_{i}: i=0,1,2, \ldots, N+1\right\}
\end{aligned}
$$

onde $h$ é o tamanho de passo espacial, e $N+1$ e $x_{i}$ são o número de partições e os pontos de $\bar{\Omega}_{h}$, respectivamente. $\Omega_{h}$ é o domínio espacial discretizado, e $\partial \bar{\Omega}_{h}$ é formado por $x_{0}=0$ e $x_{N+1}=L$. 
Número de iterações espaciais: $N+1=(L-0) / h$.

\subsubsection{Discretização temporal}

Defina-se:

$$
\begin{aligned}
\tau & =\left(T_{\max }-0\right) /(M+1), \quad t_{n}=0+n \tau, \quad n=0,1,2, \ldots, M+1 \\
\bar{I}^{\tau} & =\left\{t_{n}: n=0,1,2, \ldots, M+1\right\}
\end{aligned}
$$

onde $\tau$ é o tamanho de passo temporal, $M+1$ e $t_{n}$ são o número de partições e os pontos de $\bar{I}^{\tau}$, respectivamente, $\bar{I}^{* \tau}$ é o domínio temporal discretizado e $\partial \bar{I}^{\tau}$ está dada pelos pontos: $t_{0}=0$ e $t_{M+1}=T_{\max }$, em que $t_{0}=0$ representa o instante inicial.

Número de iterações temporais: $M+1=T_{\max } / \tau$.

O domínio espaço-temporal discreto do problema é definido por $Q_{h}^{\tau}=\bar{\Omega}_{h} \times \bar{I}^{\tau}$. Na Figura 4 mostramos este domínio.

Figura 4 - Domínio espaço-temporal discreto, $Q_{h}^{\tau}$.

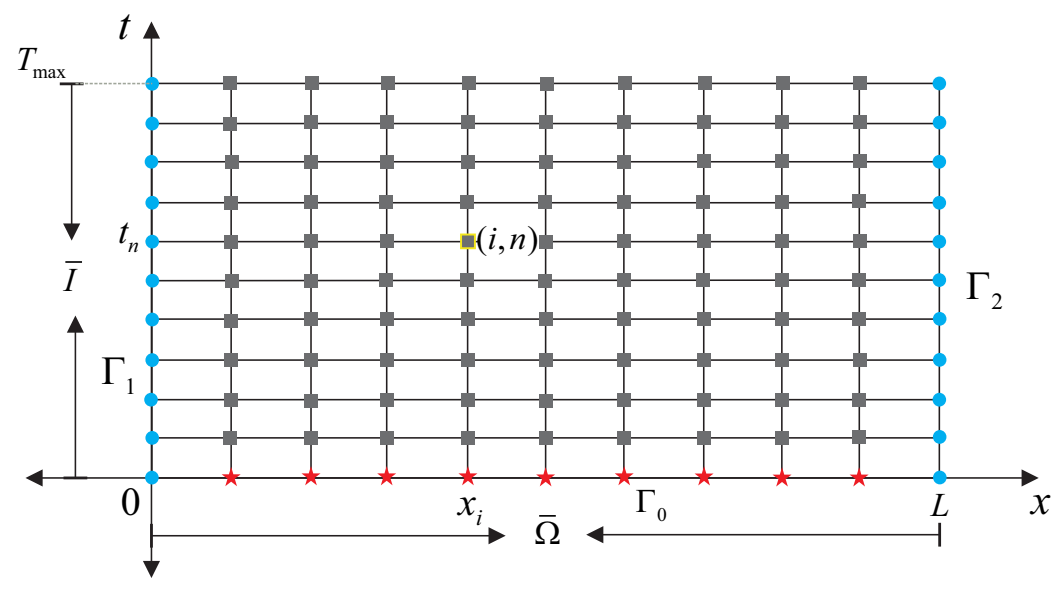

Fonte: Elaboração dos autores (2020).

As linhas-verticais discretas, denotas por "círculos cheios",

$$
\Gamma_{1}=\left\{\left(0, t_{n}\right): t_{n} \in \bar{I}^{\tau}\right\} \quad \text { e } \quad \Gamma_{2}=\left\{\left(L, t_{n}\right): t_{n} \in \bar{I}^{\tau}\right\}
$$

correspondem à condição de contorno. A linha-horizontal discreta, denotada por "asteriscos",

$$
\Gamma_{0}=\left\{\left(x_{i}, 0\right): x_{i} \in \Omega_{h}\right\}
$$

corresponde à condição inicial. Em cada nó dessa linha, a função-incógnita assume seus valores iniciais. Finalmente, o retângulo discreto, denotado por "quadradinhos cheios", denota o domínio 
espaço-temporal discreto interno. Em cada nó desse retângulo, a função-incógnita será determinada.

\subsection{Método de Diferenças Finitas}

Uma vez discretizado o domínio espaço-temporal, procedemos a encontrar a solução aproximada do problema. O método de aproximação a ser utilizado é o método de Diferenças Finitas, mais especificamente, o Método de Crank-Nicolson, que é implícito no tempo, possui segunda ordem de convergência no espaço e tempo, e é numericamente estável.

\subsubsection{Método de Crank-Nicolson}

A função $u(x, t)$ e suas derivadas são aproximadas pelos seguintes operadores discretos:

$$
\begin{aligned}
u\left(x_{i}, t_{n}\right) & =u_{i}^{n} \\
\left(u_{t}\right)_{i}^{n} & \approx \delta_{t}^{+} u_{i}^{n}:=\left(u_{i}^{n+1}-u_{i}^{n}\right) / \tau \quad \text { (Diferença para frente, no tempo) } \\
\left(u_{x}\right)_{i}^{n} & \approx \delta_{x}^{0} u_{i}^{n}:=\left(u_{i+1}^{n}-u_{i-1}^{n}\right) / 2 h \quad \text { (Diferença central, no espaço) } \\
\left(u_{x x}\right)_{i}^{n} & \approx \delta_{x x}^{0} u_{i}^{n}:=\left(u_{i+1}^{n}-2 u_{i}^{n}+u_{i-1}^{n}\right) / h^{2} \quad \text { (Diferença central de } 2^{\mathrm{a}} \text { ordem, no espaço) }
\end{aligned}
$$

Considere um problema 1D, modelado por uma EDP de segunda ordem na variável espacial e de primeira ordem na variável temporal,

$$
\frac{\partial u(x, t)}{\partial t}=f\left(x, \frac{\partial u}{\partial x}, \frac{\partial^{2} u}{\partial x^{2}}\right)+g(u)
$$

No nó $(i, n)$, o método de Crank-Nicolson é definido por:

$$
\frac{u_{i}^{n+1}-u_{i}^{n}}{\tau}=\frac{1}{2}\left(f_{i}^{n}+f_{i}^{n+1}\right)+\frac{1}{2}\left(g\left(u_{i}^{n}\right)+g\left(u_{i}^{n+1}\right)\right)
$$

onde o tamanho de passo espacial pode ser $h, h / 2, h / 4, \ldots$

\subsubsection{Discretização da equação que modela o problema}

A equação (1) é discretizada pelo Método de Crank-Nicolson. Como (1) é um caso particular de (11), temos de (12) que

$$
\frac{c_{i}^{n+1}-c_{i}^{n}}{\tau}=\frac{1}{2}\left(f_{i}^{n}+f_{i}^{n+1}\right)+\frac{1}{2}\left(g_{i}^{n}+g_{i}^{n+1}\right)
$$


onde

$$
f_{i}^{n}=\delta_{x}^{0}\left(D_{i}\left(\delta_{x}^{0} c_{i}^{n}\right)\right) \quad \text { e } g_{i}^{n}=r c_{i}^{n}\left(1-c_{i}^{n} / \kappa_{i}\right)
$$

A equação (13) é a equação discretizada de nosso problema.

Como o coeficiente de difusão $D(x)$ depende da variável espacial, LeVeque (2007, p. 36) considera mais vantajoso discretizar o termo $f_{i}^{n}$, de [14], diretamente, preservando a física do problema, e menos vantajoso quando se aplica, primeiro, a regra da cadeia para depois discretizar. Em nosso trabalho, ainda que $D(x)$ seja descontínuo, temos aplicado a ideia mais vantajosa.

Consideremos no nó $(i, n)$ aproximações com tamanho de passo $h / 2$, sendo $h=x_{i+1}-x_{i}$. Assim, de (14),

$$
\begin{aligned}
f_{i}^{n} & =\delta_{x}^{0}\left(D_{i}\left(c_{i+1 / 2}^{n}-c_{i-1 / 2}^{n}\right) / h\right)=\left(D_{i+1 / 2} \frac{c_{i+1}^{n}-c_{i}^{n}}{h}-D_{i-1 / 2}^{n} \frac{c_{i}^{n}-c_{i-1}^{n}}{h}\right) / h \\
& =\left(1 / h^{2}\right)\left(D_{i+1 / 2} c_{i+1}^{n}-\left(D_{i+1 / 2}+D_{i-1 / 2}\right) c_{i}^{n}+D_{i-1 / 2} c_{i-1}^{n}\right)
\end{aligned}
$$

e

$$
g_{i}^{n}=r c_{i}^{n}\left(\kappa_{i}-c_{i}^{n}\right) / \kappa_{i}
$$

Denote, $q_{i}^{n}=\frac{r}{\kappa_{i}}$. Então, 16 é dado por

$$
g_{i}^{n}=q_{i}^{n}\left(\kappa_{i}-c_{i}^{n}\right) c_{i}^{n}
$$

Rearranjando termos em (13), temos

$$
c_{i}^{n+1}-(\tau / 2) f_{i}^{n+1}-(\tau / 2) g_{i}^{n+1}=c_{i}^{n}+(\tau / 2) f_{i}^{n}+(\tau / 2) g_{i}^{n}
$$

Para o lado esquerdo de (18),

$$
\begin{aligned}
c_{i}^{n+1}-(\tau / 2) f_{i}^{n+1}-(\tau / 2) g_{i}^{n+1} & =c_{i}^{n+1}-\lambda\left(D_{i+1 / 2} c_{i+1}^{n+1}-\left(D_{i+1 / 2}+D_{i-1 / 2}\right) c_{i}^{n+1}+D_{i-1 / 2} c_{i-1}^{n+1}\right) \\
& -(\tau / 2) q_{i}^{n+1}\left(\kappa_{i}-c_{i}^{n+1}\right) c_{i}^{n+1} \\
& =-\lambda D_{i-1 / 2} c_{i-1}^{n+1} \\
& +\left[1+\lambda\left(D_{i+1 / 2}+D_{i-1 / 2}\right)-\frac{\tau}{2} q_{i}^{n+1}\left(\kappa_{i}-c_{i}^{n+1}\right)\right] c_{i}^{n+1}-\lambda D_{i+1 / 2} c_{i+1}^{n+1} \\
& =\alpha_{1 i} c_{i-1}^{n+1}+\alpha_{2 i}^{n}\left(c_{i}^{n+1}\right) c_{i}^{n+1}+\alpha_{3 i} c_{i+1}^{n+1}
\end{aligned}
$$

onde

$$
\begin{aligned}
\lambda & =\tau /\left(2 h^{2}\right), \quad \alpha_{1 i}=-\lambda D_{i-1 / 2} \\
\alpha_{2 i}\left(c_{i}^{n+1}\right) & =1+\lambda\left(D_{i+1 / 2}+D_{i-1 / 2}\right)-(\tau / 2) q_{i}^{n+1}\left(\kappa_{i}-c_{i}^{n+1}\right) \quad \text { e } \quad \alpha_{3 i}=-\lambda D_{i+1 / 2}
\end{aligned}
$$


De forma análoga, para o lado direito de (18), temos

$$
\begin{aligned}
c_{i}^{n}+\frac{\tau}{2} f_{i}^{n}+\frac{\tau}{2} g_{i}^{n} & =c_{i}^{n}+\lambda\left(D_{i+1 / 2} c_{i+1}^{n}-\left(D_{i+1 / 2}+D_{i-1 / 2}\right) c_{i}^{n}+D_{i-1 / 2} c_{i-1}^{n}\right)+\frac{\tau}{2} q_{i}^{n}\left(\kappa_{i}-c_{i}^{n}\right) c_{i}^{n} \\
& =\lambda D_{i-1 / 2} c_{i-1}^{n}+\left[1-\lambda\left(D_{i+1 / 2}+D_{i-1 / 2}\right)+\frac{\tau}{2} q_{i}^{n}\left(\kappa_{i}-c_{i}^{n}\right)\right] c_{i}^{n}+\lambda D_{i+1 / 2} c_{i+1}^{n} \\
& =\beta_{1 i} c_{i-1}^{n}+\beta_{2 i}^{n}\left(c_{i}^{n}\right) c_{i}^{n}+\beta_{3 i} c_{i+1}^{n}
\end{aligned}
$$

onde

$$
\beta_{1 i}=\lambda D_{i-1 / 2}, \quad \beta_{2 i}\left(c_{i}^{n}\right)=1-\lambda\left(D_{i+1 / 2}+D_{i-1 / 2}\right)+(\tau / 2) q_{i}^{n}\left(\kappa_{i}-c_{i}^{n}\right) \quad \text { e } \quad \beta_{3 i}=\lambda D_{i+1 / 2}
$$

Substituindo (19) e 21) em 18, e para cada $n=0,1,2, \ldots, M$ e para $i=1,2,3, \ldots, N$, temos

$$
\alpha_{1 i} c_{i-1}^{n+1}+\alpha_{2 i}\left(c_{i}^{n+1}\right) c_{i}^{n+1}+\alpha_{3 i} c_{i+1}^{n+1}=\beta_{1 i} c_{i-1}^{n}+\beta_{2 i}\left(c_{i}^{n}\right) c_{i}^{n}+\beta_{3 i} c_{i+1}^{n}
$$

A equação (23) representa um sistema algébrico não-linear que resultou da equação (13).

\subsubsection{Condições de contorno}

Condições de contorno Tipo Neumann

$$
\begin{aligned}
& \frac{\partial u}{\partial x}(0, t)=\phi(t) \\
& \frac{\partial u}{\partial x}(L, t)=\psi(t)
\end{aligned}
$$

Uma aproximação para as condições de Neumann são:

Em (24), diferenças para frente

$$
\left(u_{1}^{n}-u_{0}^{n}\right) / h=\phi^{n} \Longleftrightarrow u_{0}^{n}=u_{1}^{n}-h \phi^{n}
$$

Em (25), diferenças para trás

$$
\left(u_{N+1}^{n}-u_{N}^{n}\right) / h=\psi^{n} \Longleftrightarrow u_{N+1}^{n}=u_{N}^{n}+h \psi^{n}
$$

Em nosso problema, as condições de contorno de tipo Neumann (4) são discretizadas como

$$
u_{0}^{n}=u_{1}^{n} \text { e } \quad u_{N+1}^{n}=u_{N}^{n}
$$




\subsubsection{Modelo matricial}

A equação (23), em termos matriciais, pode ser expressa por

$$
\left[\begin{array}{cccccc}
\alpha_{21}\left(c_{1}^{n+1}\right) & \alpha_{31} & 0 & 0 & \ldots & 0 \\
\alpha_{12} & \alpha_{22}\left(c_{2}^{n+1}\right) & \alpha_{32} & 0 & \cdots & 0 \\
0 & \alpha_{13} & \alpha_{23}\left(c_{3}^{n+1}\right) & \alpha_{33} & \cdots & 0 \\
\vdots & \ddots & \ddots & \ddots & \ldots & \vdots \\
0 & \ldots & 0 & \alpha_{1(N-1)} & \alpha_{2(N-1)}\left(c_{N-1}^{n+1}\right) & \alpha_{3(N-1)} \\
0 & \ldots & 0 & 0 & \alpha_{1 N} & \alpha_{2 N}\left(c_{N}^{n+1}\right)
\end{array}\right]\left[\begin{array}{c}
c_{1}^{n+1} \\
c_{2}^{n+1} \\
c_{3}^{n+1} \\
\vdots \\
c_{N-1}^{n+1} \\
c_{N}^{n+1}
\end{array}\right]
$$$$
\left[\begin{array}{cccccc}
\beta_{21}\left(c_{1}^{n}\right) & \beta_{31} & 0 & 0 & \ldots & 0 \\
\beta_{12} & \beta_{22}\left(c_{2}^{n}\right) & \beta_{32} & 0 & \ldots & 0 \\
0 & \beta_{13} & \beta_{23}\left(c_{3}^{n}\right) & \beta_{33} & \cdots & 0 \\
\vdots & \ddots & \ddots & \ddots & \ldots & \vdots \\
0 & \ldots & 0 & \beta_{1(N-1)} & \beta_{2(N-1)}\left(c_{N-1}^{n}\right) & \beta_{3(N-1)} \\
0 & \cdots & 0 & 0 & \beta_{1 N} & \beta_{2 N}\left(c_{N}^{n}\right)
\end{array}\right]\left[\begin{array}{c}
c_{1}^{n} \\
c_{2}^{n} \\
c_{3}^{n} \\
\vdots \\
c_{N-1}^{n} \\
c_{N}^{n}
\end{array}\right]+\left[\begin{array}{c}
\beta_{11} c_{0}^{n} \\
0 \\
\vdots \\
0 \\
\beta_{3 N} c_{N+1}^{n}
\end{array}\right]
$$

Denote

$$
\mathbf{c}=\left(c_{1}, c_{2}, c_{3}, \ldots, c_{N}\right)^{T}
$$

No nível $(n+1)$, tem-se:

$$
\begin{aligned}
& \mathrm{A}(\mathbf{c})=\left(a_{i j}(\mathbf{c})\right)_{N \times N}=\left\{\begin{array}{rll}
\alpha_{1 i}, & \text { se } & j=i-1 \\
\alpha_{2 i}\left(c_{i}\right), & \text { se } & j=i \\
\alpha_{3 i}, & \text { se } & j=i+1
\end{array}\right. \\
& \mathbf{v}_{1}=\left(v_{1 i}\right)_{N \times 1}=\left(\alpha_{11} c_{0}, 0, \ldots, 0, \alpha_{3 N} c_{N+1}\right)^{T}
\end{aligned}
$$

No nível $(n)$, tem-se:

$$
\begin{aligned}
\mathrm{B}(\mathbf{c}) & =\left(\tilde{b}_{i j}(\mathbf{c})\right)_{N \times N}=\left\{\begin{array}{ccc}
\beta_{1 i}, & \text { se } & j=i-1 \\
\beta_{2 i}\left(c_{i}\right), & \text { se } & j=i \\
\beta_{3 i}, & \text { se } & j=i+1
\end{array}\right. \\
\mathbf{v}_{0} & =\left(v_{0 i}\right)_{N \times 1}=\left(\beta_{11} c_{0}, 0, \ldots, 0, \beta_{3 N} c_{N+1}\right)^{T}
\end{aligned}
$$


Assim, 27] pode ser expressa, na sua forma matricial compacta, por

$$
\mathrm{A}(\mathbf{c}) \mathbf{c}+\mathbf{v}_{1}=\mathrm{B}(\mathbf{c}) \mathbf{c}+\mathbf{v}_{0}
$$

Como o problema apresenta condições de contorno de tipo Neumann, é preciso aproximar os vetores $\mathbf{v}_{1}$ e $\mathbf{v}_{0}$. De 26, 27) e 28, segue que

$$
\mathbf{v}_{1}=\left[\begin{array}{c}
\alpha_{11} c_{0}^{n+1} \\
0 \\
\vdots \\
0 \\
\alpha_{3 N} c_{N+1}^{n+1}
\end{array}\right]_{N \times 1}=\left[\begin{array}{c}
\alpha_{11}\left(c_{1}^{n+1}-h \phi^{n+1}\right) \\
0 \\
\vdots \\
0 \\
\alpha_{3 N}\left(c_{N}^{n+1}+h \psi^{n+1}\right)
\end{array}\right]=\left[\begin{array}{ccccc}
\alpha_{11} & 0 & \ldots & 0 & 0 \\
0 & 0 & \ldots & 0 & 0 \\
\vdots & \vdots & \ddots & \vdots & \vdots \\
0 & 0 & \ldots & 0 & 0 \\
0 & 0 & \ldots & 0 & \alpha_{3 N}
\end{array}\right]_{N \times N}\left[\begin{array}{c}
c_{1}^{n+1} \\
0 \\
\vdots \\
0 \\
c_{N}^{n+1}
\end{array}\right]
$$

Essa última equação, em sua forma matricial compacta, é dada por

$$
\mathbf{v}_{1}^{n+1}=\mathrm{D}_{1} \mathbf{c}^{n+1}
$$

onde

$$
\mathrm{D}_{1}=\left(d_{1 i i}\right)_{N \times N}=\operatorname{diag}\left\{\alpha_{11}, 0, \ldots, 0, \alpha_{3 N}\right\}=\left\{\begin{array}{rll}
\alpha_{11}, & \text { se } & i=1 \\
0, & \text { se } & 1<i<N \\
\alpha_{3 N}, & \text { se } & i=N
\end{array}\right.
$$

Procedemos de forma análoga com o vetor $\mathbf{v}_{0}$. Assim,

$$
\mathbf{v}_{0}^{n}=\mathrm{D}_{0} \mathbf{c}^{n}
$$

onde

$$
\mathrm{D}_{0}=\left(d_{0 i i}\right)_{N \times N}=\operatorname{diag}\left\{\beta_{11}, 0, \ldots, 0, \beta_{3 N}\right\}=\left\{\begin{array}{rll}
\beta_{11}, & \text { se } & i=1 \\
0, & \text { se } & 1<i<N \\
\beta_{3 N}, & \text { se } & i=N
\end{array}\right.
$$

Levando em consideração as condições de contorno, a equação (27) é dada por

$$
\mathrm{A}\left(\mathbf{c}^{n+1}\right) \mathbf{c}^{n+1}+\mathrm{D}_{1} \mathbf{c}^{n+1}=\mathrm{B}\left(\mathbf{c}^{n}\right) \mathbf{c}^{n}+\mathrm{D}_{0} \mathbf{c}^{n}
$$

para todo $n=0,1,2,3, \ldots, M$.

Sem perda de generalidade, a equação 33 também pode expressar-se como

$$
\mathcal{M}(\mathbf{c}) \mathbf{c}=\mathbf{b}
$$


onde

$$
\mathcal{M}(\mathbf{c})=\mathrm{A}\left(\mathbf{c}^{n+1}\right)+\mathrm{D}_{1}
$$

e

$$
\mathbf{b}=\mathrm{B}\left(\mathbf{c}^{n}\right) \mathbf{c}^{n}+\mathrm{D}_{0} \mathbf{c}^{n}, \quad n=0,1,2,3, \ldots, M
$$

\section{Solução numérica}

Nesta seção, apresentaremos o sistema algébrico não-linear, que resultou da discretização da equação que modela o problema. Esse sistema será resolvido pelo Método de Newton.

\subsection{Solução do sistema algébrico não-linear}

A equação (34) pode expressar-se como um sistema algébrico não-linear, isto é,

$$
\mathbf{f}(\mathbf{c})=\mathcal{M}(\mathbf{c}) \mathbf{c}-\mathbf{b}=0
$$

ou, equivalentemente,

$$
f_{i}(\mathbf{c})=\sum_{j=1}^{N} m_{i j}(\mathbf{c}) c_{j}-b_{i}, \quad i=1,2,3, \ldots, N
$$

O sistema (37), com solução inicial, é formulado por

$$
\left\{\begin{array}{c}
\mathbf{f}(\mathbf{c})=0 \\
\mathbf{c}^{0} \in \mathbb{R}^{N}
\end{array}\right.
$$

Utilizaremos o Método de Newton para resolver (39). Nesse caso, a equação iterativa é dada por

$$
\begin{aligned}
J \mathbf{f}\left(\mathbf{c}^{k-1}\right) \mathbf{d}^{k} & =-\mathbf{f}\left(\mathbf{c}^{k-1}\right), \quad k=1,2,3, \ldots \\
\mathbf{c}^{k} & =\mathbf{c}^{k-1}+\mathbf{d}^{k}
\end{aligned}
$$

onde $\mathbf{f}(\mathbf{c})$ é dado por 38 e a matriz jacobiana, Jf(c), é dada por

$$
J \mathbf{f}(\mathbf{c})=\left(\frac{\partial f_{i}(\mathbf{c})}{\partial c_{j}}\right)_{N \times N}
$$


onde

$$
\frac{\partial f_{i}(\mathbf{c})}{\partial c_{j}}=\left\{\begin{array}{rll}
\alpha_{1 i}, & \text { se } & j=i-1 \\
(\tau / 2) q_{i}^{n+1} c_{i}+\alpha_{2 i}\left(c_{i}\right)+d_{1 i i}, & \text { se } & j=i \\
\alpha_{3 i}, & \text { se } & j=i+1
\end{array}\right.
$$

Note que em (40) resolve-se um sistema linear cada vez que $k$ assume um valor, e com a equação (41) obtemos a solução de nosso problema. Finalmente, depois de um certo número de iterações, que depende da solução inicial e do critério de parada, encontramos a solução numérica de nosso problema.

Para a obtenção da solução numérica foi implementado, na Linguagem Fortran, o sistema não-linear discretizado (37). Os compiladores utilizados foram: (a) gfortran - gnu fortran compiler e (b) compilador online (https://www.onlinegdb.com/).

\section{Resultados e discussão}

Nesta seção, apresentaremos e explicaremos os resultados numéricos da concentração de células do glioma, e determinaremos a quantidade média de células tumorais no seu microambiente. Os resultados numéricos baseiam-se na execução de dois testes.

\subsection{Concentração de células tumorais}

Para a simulação da dinâmica do glioma, foram considerados dois testes; para cada um deles estudou-se dois modelos:

- Modelo 1: Concentração de células do glioma, considerando a função capacidade de carga constante, ou seja, quando $a=c_{\max }=62,5$, conforme Özuğurlu (2015).

- Modelo 2: Concentração de células do glioma, considerando a função capacidade de carga variável, sendo $a=0$.

Cada modelo precisa dos seguintes parâmetros: $L$, o comprimento do microambiente tumoral, $r$, a taxa de proliferação do glioma, $a$, a constante não-negativa da função capacidade de carga, $h$, o tamanho de passo espacial, $\tau$, o tamanho de passo temporal e $T_{\max }$, o tempo máximo da simulação. 


\subsubsection{Teste 1}

O Teste 1 refere-se ao $1^{\circ}, 5^{\circ}, 10^{\circ}, 20^{\circ}, 30^{\circ}$ e $60^{\circ}$ dia de simulação da dinâmica do glioma. Os parâmetros desse teste estão discriminados na Tabela 1.

Tabela 1 - Parâmetros do Teste 1.

\begin{tabular}{l||cccccc}
\hline Teste 1 & $L[\mathrm{~mm}]$ & $r[1 / \mathrm{dia}]$ & $a[$ células $/ \mathrm{mm}]$ & $h[\mathrm{~mm}]$ & $\tau[\mathrm{dia}]$ & $T_{\max }[\mathrm{dia}]$ \\
\hline \hline Modelo 1 & 50 & 0,012 & 62,5 & 0,5 & 0,2 & \multirow{2}{*}{$1,5,10,20,30$ e 60} \\
\hline Modelo 2 & 50 & 0,012 & 0 & 0,5 & 0,2 & \\
\hline
\end{tabular}

Fonte: Elaboração dos autores (2020).

Os resultados numéricos desse teste, para os modelos 1 e 2, foram plotados conforme mostram as figuras 5(a) e 5(b), respectivamente. Ambas representam os gráficos da concentração de células do glioma em função da posição $x$ no microambiente tumoral, para diferentes instantes de tempo. Percebe-se que, desde seu estado inicial até o sexagésimo dia, a concentração de células do glioma não apresenta mudança significativa quando comparados os dois modelos.

Figura 5 - Resultados do Teste 1 - Concentração de células do glioma no $1^{\circ}, 6^{\circ}, 10^{\circ}, 20^{\circ}, 30^{\circ}$ e $60^{\circ}$ dia: (a) Modelo 1 e (b) Modelo 2.

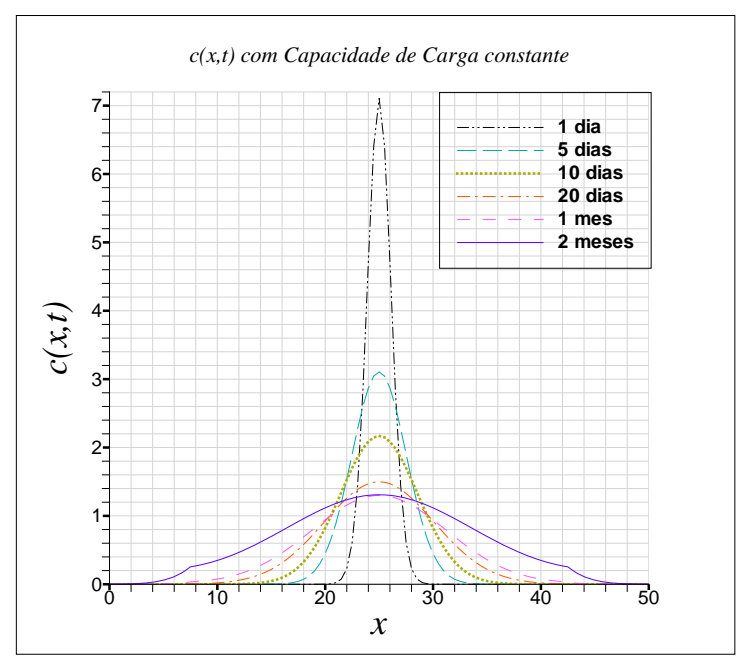

(a)

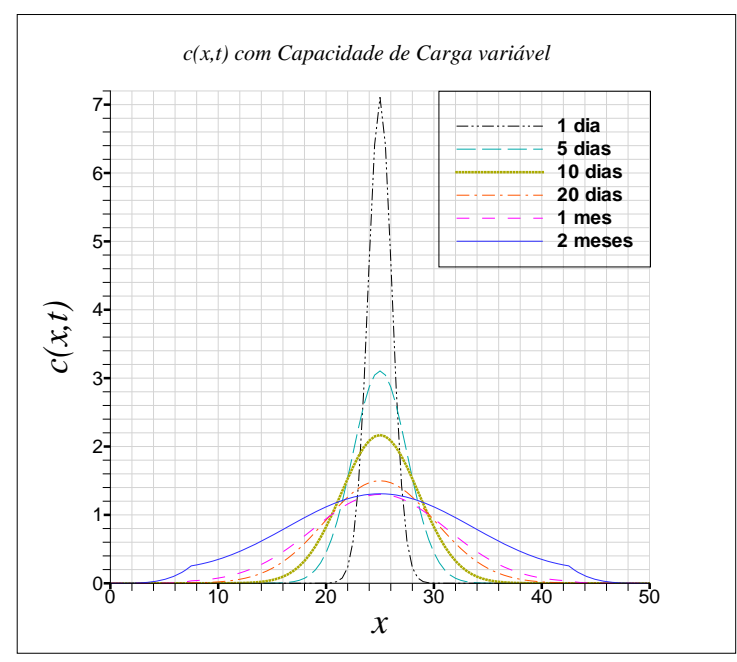

(b)

Fonte: Elaboração dos autores (2020). 


\subsubsection{Teste 2}

O Teste 2 refere-se ao $3^{\circ}, 6^{\circ}, 12^{\circ}, 18^{\circ}, 24^{\circ}, 30^{\circ}$ e $36^{\circ}$ mês de simulação da concentração de células do glioma. Os parâmetros desse teste estão discriminados na Tabela2.

Tabela 2 - Parâmetros do Teste 2.

\begin{tabular}{l||cccccc}
\hline Teste 2 & $L[\mathrm{~mm}]$ & $r[1 / \mathrm{dia}]$ & $a$ [células $/ \mathrm{mm}]$ & $h[\mathrm{~mm}]$ & $\tau[\mathrm{dia}]$ & $T_{\max }[\mathrm{meses}]$ \\
\hline \hline Modelo 1 & 50 & 0,012 & 62,5 & 0,5 & 0,2 & \multirow{2}{*}{$3,6,12,18,24,30$ e 36} \\
\hline Modelo 2 & 50 & 0,012 & 0 & 0,5 & 0,2 & \\
\hline
\end{tabular}

Fonte: Elabração dos autores (2020).

Foram plotados os resultados numéricos do Teste 2, correspondentes aos modelos 1 e 2, nas figuras 6(a) e 6(b), respectivamente. Ambas representam os gráficos da concentração de células do glioma em função da posição $x$ no microambiente tumoral, para diferentes instantes de tempo. No terceiro mês, observa-se que o glioma, ainda, continua tendo a mesma concentração em ambos os modelos. Porém, a partir do sexto mês, a concentração de células do glioma aumenta de forma considerável, em cada modelo, mas de forma diferente, quando comparados os modelos. Os gráficos da concentração de células do glioma do Modelo 1, veja Figura 6(a), crescem em forma de linhas retas horizontais até atingir o estado estacionário após 3 anos, coincidindo com os gráficos da concentração de células do glioma estudado por Özuğurlu (2015, p. 1510). Por outro lado, os gráficos da concentração de células do glioma do Modelo 2, veja Figura 6(b), crescem, ao longo do tempo, em forma de parábolas invertidas cada vez fechando-se mais, mas levemente, à medida que se atinge o estado estacionário do glioma, ou seja, após 3 anos. No Modelo 1, observa-se, também, que, para valores de $t$ suficientemente grandes, a concentração $c(x, t)$ atinge o seu valor máximo, isto é, $\lim _{t \rightarrow \infty} c(x, t)=c_{\max }=62,5$, enquanto que, no Modelo 2, não acontece.

\subsection{Quantidade média de células tumorais}

Os dois testes anteriores proporcionaram a dinâmica do crescimento do glioma no seu microambiente. Precisamos, agora, medir esse crescimento e, para esse fim, criou-se a função quantidade média de células tumorais. 
Figura 6 - Resultados do Teste 2 - Concentração de células do glioma no $3^{\circ}, 6^{\circ}, 12^{\circ}, 18^{\circ}, 24^{\circ}, 30^{\circ}$ e $36^{\circ}$ mês: (a) Modelo 1 e (b) Modelo 2.

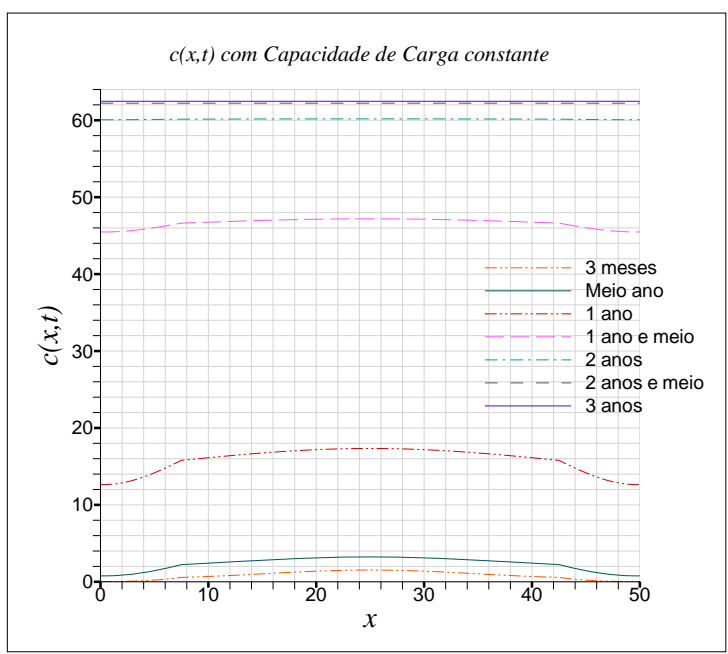

(a)

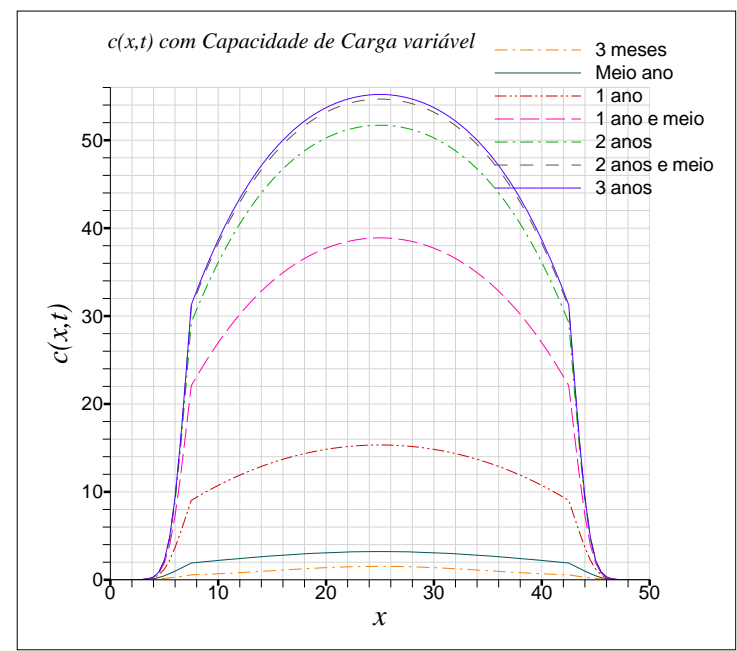

(b)

Fonte: Elaboração dos autores (2020).

Definição 5.1 (Função Quantidade Média de Células Tumorais). Considere-se um microambiente tumoral 1D e de comprimento $L$. Define-se a função quantidade média de células tumorais, no seu microambiente e no instante de tempo $t$, por

$$
Q(t)=\int_{0}^{L} u(x, t) d x
$$

onde $u(x, t)$ determina a quantidade de células tumorais, por unidade de milímetro, na posição $x \mathrm{e}$ no instante de tempo $t$, de seu microambiente.

Na Figura 7(a) observamos oito gráficos da função $Q(t)$ para diferentes valores de $a=$ $0,10,20,30,40,50,60,62,5$, em $\kappa(x)$. Observamos que à medida que aumenta o parâmetro $a$, de 0 até 62,5 , a quantidade média de células do glioma, no microambiente tumoral, aumenta. Por exemplo, quando $a=c_{\max }=62,5$ (Modelo 1), o valor máximo de $Q(t)$ ocorre em $t=1080$ dias, e a quantidade média de células do glioma é $Q(1080)=3123$ células, sendo o valor exato $c_{\max } L=3125$ células. Também, $Q(1080)=1752$ células quando $a=0$ (Modelo 2 ), mas o valor exato é $\int_{0}^{50} \kappa(x) d x=2188,94$ células, o que significa que, no regime permanente, a concentração de células do glioma não converge à sua capacidade de carga. Inicialmente, a quantidade média de células do glioma, no microambiente tumoral, foi $Q(0)=\int_{0}^{50} c_{0}(x) d x=1$ célula. Para determinar, exatamente, no que se refere ao aumento da quantidade média de células tumorais do Modelo 1 em 
relação ao Modelo 2, calculamos o erro relativo, dado pela seguinte fórmula:

$$
E_{r}(t)=\frac{\left|Q_{M_{1}}(t)-Q_{M_{2}}(t)\right|}{\left|Q_{M_{1}}(t)\right|}
$$

onde $Q_{M_{1}}$ e $Q_{M_{2}}$ são as quantidades médias de células do glioma, ao longo do tempo, do Modelo 1 e Modelo 2, respectivamente. Na Figura 7(b) mostramos o gráfico do erro relativo (dado em porcentagem), desde o instante inicial até os 1080 dias. Pode ser observado que, no primeiro ano, o glioma é $38,3 \%$ maior, no Modelo 1 que no Modelo 2, no segundo ano $45,4 \%$ e, no regime permanente, $43,9 \%$.

Figura 7 - (a) Quantidade média de células do glioma, no microambiente tumoral, ao longo do tempo. Foram considerados oito casos para diferentes valores de $a$, da função capacidade de carga. (b) Erro relativo, ao longo do tempo. Os valores no eixo das ordenadas são dados em porcentagem.

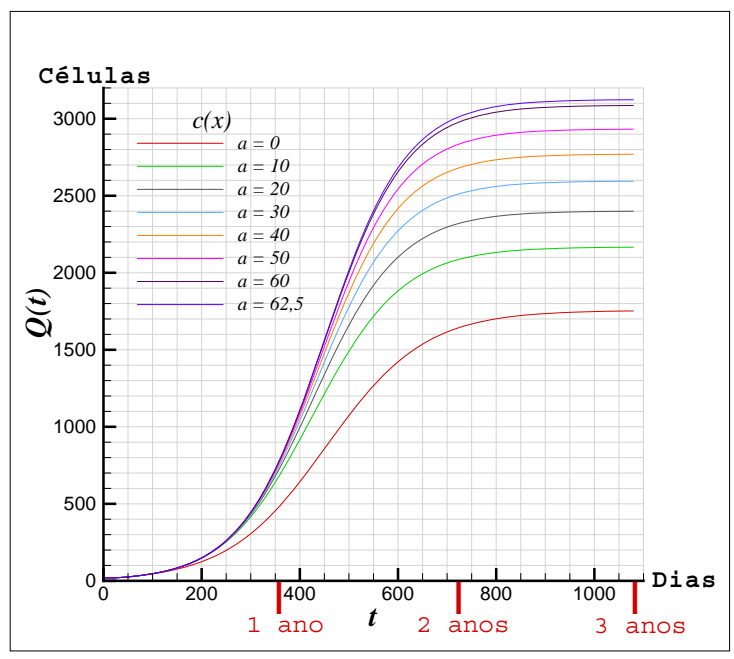

(a)

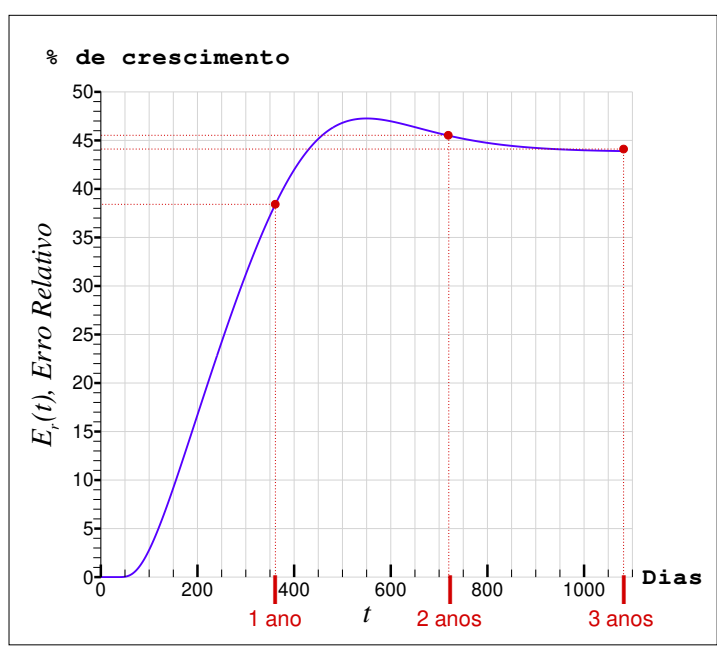

(b)

Fonte: Elaboração dos autores (2020).

\section{Considerações finais}

Foi resolvido numericamente, pelo método de Crank-Nicolson, o problema da dinâmica do crescimento de um glioma. Por considerar-se a natureza difusiva do glioma, a presença física de seu microambiente é indispensável, ou seja, é necessário introduzir a variável espacial no problema. Por simplicidade, foi estudado o problema 1D. A presença da variável espacial repercute, principalmente, no termo difusivo da ED-R, mais especificamente, no coeficiente de difusão, que em nosso caso foi considerado descontínuo por causa da heterogeneidade do microambiente tumoral, mas 
esse fato já foi estudado por Özuğurlu (2015). Por outro lado, considerou-se, no termo da taxa líquida de proliferação, da ED-R, o modelo de crescimento logístico com capacidade de carga variando espacialmente. Esse fato é novo com relação ao tema da dinâmica do crescimento de gliomas difusivos com coeficientes de difusão descontínuos, pois, na maioria dos casos, assumem o microambiente tumoral homogêneo ou relativamente uniforme, dessa forma, a capacidade de carga é considerada constante. A ideia nasce ao perceber-se a heterogeneidade do microambiente tumoral. Deangelis et al. (2020) estudaram a capacidade de carga dependente da variável espacial, em dinâmica populacional em ecologia. Foi assumido, o perfil da capacidade de carga como sendo o gráfico de uma Função de Hill, pela mesma razão que uma onda retangular representa o coeficiente de difusão, com a diferença que o perfil é contínuo, e a onda não. Os resultados apontam que, no regime permanente, a quantidade média de células do glioma é $44 \%$ maior no Modelo 1 do que no Modelo 2. Desse modo, concluímos que o crescimento do glioma é menos afetado quando a capacidade de carga é considerada variável do que constante. Finalmente, esperamos que estes resultados possam contribuir no prognóstico de pacientes com glioma, mas certamente contribuirão no desenvolvimento da oncologia matemática.

\section{Agradecimento}

À Fundação de Amparo à Pesquisa do Estado de Minas Gerais (FAPEMIG) por ter financiado o Projeto submetido ao Programa Institucional de Bolsas de Iniciação Científica, PIBIC/UFSJ, que deu origem a esse artigo.

\section{Referências}

AVILA, J. A. J.; LOZADA-CRUZ, G. J. On a Model for the Growth of an Invasive Avascular Tumor. Applied Mathematics \& Information Sciences, v. 7, n. 5, p. 1857-1863, 2013. DOI: http://dx.doi.org/10.12785/amis/070524.

BONILLA, L. L.; CAPASSO, V.; ALVARO, M.; CARRETERO, M.; TERRAGNI, F. On the Mathematical Modelling of Tumor-induced Angiogenesis. Mathematical Biosciences and Engineering, v. 14, n. 1, p. 45-66, 2017. DOI: http://dx.doi.org/10.3934/mbe.2017004.

CHAPLAIN, M. A.; SLEEMAN, B. D. A mathematical model for the production and secretion of tumour angiogenesis factor in tumours. IMA Journal of Mathematics Applied in Medicine and Biology, v. 7, n. 2, p. 93-108, 1990. DOl:https://doi.org/10.1093/imammb/7.2.93.

CRUYWAGEN, G. C.; WOODWARD, D. E.; TRACQUI, P.; BARTOO, G. T.; MURRAY, J. D.; ALVORD, E. C. The Modelling of Diffusive Tumours. Journal of Biological Systems, v. 3, n. 4, p. 
937-945, 1995. DOI: https://doi.org/10.1142/S0218339095000836.

DEANGELIS, D. L.; ZHANG, B.; NI, W.-M.; WANG,Y. Carrying Capacity of a Population Diffusing in a Heterogeneous Environment. Mathematics, v. 8, n. 49, p. 1-12, 2020. DOI:

https://doi.org/10.3390/math8010049.

DEHGHAN, M.; MOHAMMADI, V. Comparison between two meshless methods based on collocation technique for the numerical solution of four-species tumor growth model.

Communications in Nonlinear Science and Numerical Simulation, v. 44, p. 204-219, 2017. DOI: https://doi.org/10.1016/j.cnsns.2016.07.024.

FOLKMAN, J. The vascularization of tumors. Scientific American, v. 234, p. 58-73, 1976. DOI: https://doi.org/10.1038/scientificamerican0576-58.

GARCIA, M. G.; GÓMEZ, A. H. Manual de Oncologia: Procedimientos Médicos Quirúrgicos. 4. ed., McGrawHill, 2010.

HARTUNG, N.; MOLLARD, S.; BARBOLOSI, D.; BENABDALLAH, A.; CHAPUISAT, G.; HENRY, G.; GIACOMETTI, S.; ILIADIS, A.; CICCOLINI, J.; FAIVRE, C.; HUBERT, F. Mathematical modeling of tumor growth and metastatic spreading: validation in tumor-bearing mice. Cancer Research, $v$. 74, n. 22, p. 6397-6407, 2014. DOI: https://doi.org/10.1158/0008-5472. can-14-0721.

INCA. Instituto Nacional de Câncer José Alencar Gomes da Silva. Estimativa 2020: incidência de câncer no Brasil. Rio de Janeiro: INCA, 2019. Disponível em: https://www.inca.gov.br/sites/ufu.sti.inca.local/files/media/document/ estimativa-2020-incidencia-de-cancer-no-brasil.pdf. Acesso em: 29 mar. 2020.

INSTITUTO ONCOGUIA. Tipos de Câncer: Tumores Cerebrais/Sistema Nervoso Central. Atualização 20 abr. 2018. Disponível em: http://www.oncoguia.org.br/conteudo/tipos-de-tumores-cerebrais-snc/894/293. Acesso em: 29 mar. 2020.

KIM, Y.; JEON, H.; OTHMER, H. The role of the tumor microenvironment in glioblastoma: A mathematical model. Institute of Electrical and Electronics Engineers: Transactions on Biomedical Engineering, v. 64, n. 3, p. 519-527, mar. 2017. DOI: https://dx.doi.org/10.1109\%2FTBME.2016.2637828.

KUMAR, V.; ABBAS, A. K.; ASTER, J. C. Robbins Patologia Básica. 9. ed. Filadélfia: Saunders Elsevier, 2013.

LEVEQUE, R. J. Finite Difference Methods for Ordinary and Partial Differential Equations: Steady-State and Time-Dependent Problems. Philadelphia: SIAM, 2007.

ÖZUĞURLU, E. A note on the numerical approach for the reaction-diffusion problem to model the density of the tumor growth dynamics. Computers and Mathematics with Applications, v. 69, p. 1504-1517, 2015. DOl: https://doi.org/10.1016/j. camwa.2015.04.018.

SWANSON, K. R.; ALVORD, E. C.; MURRAY, J. D. A quantitative model for differential motility of gliomas in grey and white matter. Cell Proliferation, v. 33, n. 5, p. 317-329, out. 2000. DOI: https://doi.org/10.1046/j.1365-2184.2000.00177.x. 